\title{
Bilaterale Plexuslähmung bei einem Frühgeborenen: Was ist neu?
}

\author{
Bilateral Obstetric Brachial Plexus Paralysis: What's New?
}

L. Gortner

Bibliografie

DOI 10.1055/s-0029-1192039

Klin Padiatr 2009; 221: 51

(c) Georg Thieme Verlag KG

Stuttgart · New York

ISSN 0300-8630

Korrespondenzadresse Prof. Dr. Ludwig Gortner Kliniken für Kinder- und Jugendmedizin

Universitätsklinikum des Saarlandes 66421 Homburg/Saar ludwig.gortner@uks.eu
Während die Häufigkeit der Plexusparese bei reifen Neugeborenen rückläufig ist [4] - dies ist nach aktuellen Daten der größeren Häufigkeit der Sectio caesarea bei cephalo-pelvinem Missverhältnis mit drohender Schulterdystokie zuzuschreiben [5,8] - ist die perinatal erworbene Läsion bei sehr kleinen Frühgeborenen selten, aber in den langfristigen Konsequenzen noch gravierender als bei Reifgeborenen [5].

Die bilaterale Plexuslähmung ist wesentlich seltener als die unilaterale Form, jedoch noch weitaus schwerwiegender hinsichtlich der Langzeitprognose [1].

Operative Therapieverfahren der Plexusläsionen wurden systematisch seit den 90er-Jahren des letzten Jahrhunderts eingeführt [6] und mit zunehmender Verbesserung der perioperativen Diagnostik und Therapie sowie der operativen Methoden in der laufenden Dekade zunehmend Standard [7,12].

In der vorliegenden Ausgabe der klinischen Pädiatrie berichtet die Erlanger Gruppe über die Therapieresultate der operativen Versorgung einer bilateralen Plexuslähmung bei einem sehr kleinen Frühgeborenen [3]. Die Autoren belegen eindruckvoll, dass die motorischen Funktionen nach der operativen Behandlung eklatant verbessert wurden und die motorischen Fortschritte des betroffenen Kindes als außerordentlich gut einzuschätzen sind. Obschon die exakte Pathogenese der bilateralen Störung der Plexusfunktion des Frühgeborenen nicht in der wünschenswerten Exaktheit eingegrenzt werden konnte, sind in erster Linie mechanische Faktoren zu diskutieren.

Die vorliegende Kasuistik hat zwei wesentliche Implikationen, die eines Kommentars wert sind: Vorausgesetzt die mechanische Alteration ist der wesentliche pathogenetische Faktor der vorliegenden bilateralen Plexusläsion, ist dies ein erneuter deutlicher Hinweis für die Fragilität sehr kleiner Frühgeborener. Neben den bedrohlichen Implikationen von Hirnblutungen und periventrikulären Leukomalazien hinsichtlich der akuten Morbidität und der Einschränkung langfristiger psychomotorischer Entwicklungspotenziale sehr kleiner Frühgeborener [10,11], betont diese Kasuistik den Stellenwert sehr kleiner Frühgeborener als eine relevante Risikogruppe in der Perinatologie für die Strukturen des peripheren Nervensystems, zum Teil bedingt durch mechanische perinatale Alterationen. Daher ist dem Risikofaktor der Fragilität sehr unreifer Frühgeborener sowohl in der Geburtsmedizin als auch in der Neo- natologie unter dem Aspekt der Prävention neurologischer Läsionen Rechnung zu tragen.

Weiterhin ist die Adaptation neuer, teils experimenteller Therapieformen - im vorliegenden Fall operativer Verfahren - für sehr kleine Frühgeborene ein kritischer Faktor. Aufgrund der Seltenheit der bilateralen Plexusläsionen sind naturgemäß prospektive randomisierte Studien mit adäquaten Fallzahlen schwierig umsetzbar. Andererseits sind in den genannten Bereichen Wissensgewinn und damit Fortschritte nur realisierbar, wenn diese Verläufe systematisch beschrieben, gesammelt und dokumentiert werden. Als wesentliche Messgrößen des Therapieerfolgs sind hier neben der Verbesserung der motorischen Funktionen als primäres Ziel der Therapie die Verbesserung der langfristigen Lebensqualität zu werten [2,9]. Hierzu soll die Kasuistik ein Anstoß sein.

Literatur

1 Andersen J, Watt J, Olson Jet al. Perinatal brachial plexus palsy. Paediatr Child Health 2006; 11: 93-100

2 Debling D, Spix C, Blettner $M$ et al. The cohort of longterm survivors at the German childhood cancer registry. Klin Padiatr 2008; 220: 371-377

3 Dragu A, Horch RE, Wirth $S$ et al. Bilateral obstetric brachial plexus paralysis: A case report. Klin Padiatr 2009; 221: 57-59

4 Evans-Jones G, Kay SP, Weindling AM et al. Congenital brachial palsy: incidence, causes, and outcome in the United Kingdom and Republic of Ireland. Arch Dis Child Fetal Neonatal Ed 2003; 88: F185-F189

5 Hankins GD, Clark SM, Munn MB. Cesarean section on request at 39 weeks: impact on shoulder dystocia, fetal trauma, neonatal encephalopathy, and intrauterine fetal demise. Semin Perinatol 2006; 30: 276-287

6 Hentz VR, Meyer RD. Brachial plexus microsurgery in children. Microsurgery 1991; 12: 175-185

7 MacNeely PD, Drake JM. A systematic review of brachial plexus surgery for birth-related brachial plexus injury. Pediatr Neurosurg 2003; 38: 57-62

8 Raio L, Ghezzi F, Di NE et al. Perinatal outcome of fetuses with a birth weight greater than $4500 \mathrm{~g}$ : an analysis of 3356 cases. Eur J Obstet Gynecol Reprod Biol 2003; 109: 160-165

9 Reinmuth S, Liebeskind AK, Wickmann L et al. Having children after surviving cancer in childhood or adolescence - results of a Berlin survey. Klin Padiatr 2008; 220: 159-165

10 Schlosser RL, Krackardt B, Weber J et al. Impact of preterm infants of less than 30 weeks gestation on the prevalence of special education in school beginners of a German city (Frankfurt/Main). Klin Padiatr 2008; 220: 57-60

11 Strassburg HM, Leimer S, Platz A et al. Long-term prognosis of former very and extremely preterm babies in adulthood in Germany. Klin Padiatr 2008; 220: 61-65

12 Vekris MD, Lykissas MG, Beris AE et al. Management of obstetrical brachial plexus palsy with early plexus microreconstruction and late muscle transfers. Microsurgery 2008; 28: 252-261 